Pacific

Journal of

Mathematics

SUR DES FONCTEURS SIMPLES

ISMAÏL BOURIZK

Volume $215 \quad$ No. 2

June 2004 


\title{
SUR DES FONCTEURS SIMPLES
}

\author{
ISMAÏL BOURIZK
}

\begin{abstract}
Let $k$ be a field of positive characteristic $p$. One considers some categories, whose objects are given classes of finite $p$ groups, and morphisms are given classes of $k$-virtual bisets, i.e., linear combinations of bisets with coefficients in $k$. The category of $k$-linear functors from such a category to the category of $k$-vector spaces is abelian, and one can try to classify and describe its simple objects, or its projective objects.

By specific subfunctors of the Burnside functor, which have a unique simple quotient $S_{Q, k}$, one will get some estimates on the $k$-dimension of the evaluations of these simple functors. These evaluations are equalities for abelian $p$-groups, and for such groups $P$ the result is even stronger, since it provides some explicit $k$-bases for the evaluations $S_{Q, k}(P)$.
\end{abstract}

\section{Introduction}

Le formalisme des bi-ensembles permet de donner une description uniforme des opérations d'induction, de restriction, d'inflation, de déflation, et de transport par isomorphisme. Cette description permet une interprétation des formules classiques, comme la formule de Mackey, en termes de produits de bi-ensembles.

Etant donné un anneau commutatif $k$, il est alors naturel de considérer des catégories dont les objets sont certaines classes de groupes finis, et les morphismes certaines classes de bi-ensembles $k$-virtuels, i.e., des combinaisons linéaires à coefficients dans $k$ de bi-ensembles. La catégorie des foncteurs $k$ linéaires d'une telle catégorie vers la catégorie des $k$-modules est abélienne, et on peut essayer de classifier et décrire ses objets simples ou ses objets projectifs.

En particulier, S. Bouc a étudié la catégorie dont les objets sont tous les groupes finis, et les morphismes tous les bi-ensembles virtuels, l'anneau $k$ étant un corps de caractéristique zéro (cf. [1]). La classification des facteurs simples du foncteur de Burnside dans ce cas fait apparaître la notion de " $b$-groupes", et les facteurs en question sont les foncteurs simples $S_{H, k}$, où $H$ est un "b-groupe".

Plus récemment, S. Bouc et J. Thévenaz ont décrit certains foncteurs simples $S_{P, k}$, où $P$ est un $p$-groupe fini et $k$ est un corps de caractéristique 
positive $q$ différente de $p$, définis sur la catégorie dont les objets sont les $p$-groupes finis (cf. [2]).

Dans ce travail on essayera de compléter ce dernier cas, en prenant pour $k$ un corps de caractéristique $p$. Grâce à des sous-foncteurs du foncteur de Burnside, qui ont un unique quotient simple $S_{Q, k}$, on obtiendra des estimations de la dimension sur $k$ des évaluations de ces foncteurs simples. Dans le cas où l'on se restreint aux $p$-groupes abéliens, ces estimations sont des égalités: soient $Q$ et $P$ deux $p$-groupes abéliens finis, tel que $Q$ est d'ordre au moins $p^{2}$, alors $\operatorname{dim}_{k} S_{Q, k}(P)$ est égale au nombre de sous-groupes $R$ de $P$ tels que $P / R \simeq Q$; et $\operatorname{dim}_{k} S_{1, k}(P)$ est égale au nombre de sous-groupes $R$ de $P$ tels que $|P / R| \leq p$. Le cas où $Q$ est d'ordre $p$, est donc laissé complètement ouvert.

\section{Notations et rappels}

- Un bi-ensemble $U$, est un ensemble sur lequel un groupe $G$ agit à gauche et un autre groupe $H$ agit à droite, de façon que

$$
g(x h)=(g x) h \quad \text { pour tous } g \in G, x \in U \text { et } h \in H .
$$

Soit $k$ un corps de caractéristique $p$ positive, et $\mathcal{C}_{k}$ la catégorie dont les objets sont les $p$-groupes finis et les morphismes sont les combinaisons $k$-linéaires de bi-ensembles. Soit $\mathcal{F}_{k}$ la catégorie abélienne des foncteurs $k$-linéaires de $\mathcal{C}_{k}$ dans la catégorie des $k$-espaces vectoriels.

Si $P$ est un $p$-groupe, soit $B(P)$ l'anneau de Burnside de $P$, alors la correspondance $P \longmapsto k \otimes_{\mathbb{Z}} B(P)$ est naturellement un objet de $\mathcal{F}_{k}$ (cf. [1] , Introduction).

Si $G$ et $H$ sont deux objets de $\mathcal{C}_{k}$, alors $\operatorname{Hom}_{\mathcal{C}_{k}}(H, G)$ est le produit tensoriel par $k$ du groupe de Grothendieck de la catégorie des $G$-ensembles$H$, le produit de deux morphismes étant défini par $k$-linéarité à partir du produit d'ensembles défini comme suit:

Soient $G$ et $H$ deux groupes finis. Si $L$ est un sous-groupe du produit $G \times H$ on notera $(G \times H) / L$ le bi-ensemble formé des classes $(g, h) L$ pour $(g, h) \in G \times H$, considéré comme $G$-ensemble- $H$ pour l'action

$$
x \cdot(g, h) L \cdot y=\left(x g, y^{-1} h\right) L .
$$

Soit $G^{\prime}$ un autre groupe fini, $E$ un $G$-ensemble- $H$ et $F$ un $H$-ensemble- $G^{\prime}$, on note $E \times_{H} F$ l'ensemble des orbites de $H$ par son action sur le produit $E \times F$ donnée par $h \cdot(x, y)=\left(x h^{-1}, h y\right)$. C'est un $G$-ensemble- $G^{\prime}:$ si $g \in G$ et $g^{\prime} \in G^{\prime}$, alors par définition

$$
g \cdot \overline{(x, y)} \cdot g^{\prime}=\overline{\left(g x, y g^{\prime}\right)}
$$

où $\overline{(x, y)}$ désigne l'image de $(x, y)$ dans $E \times_{H} F$.

Soit $H$ un sous-groupe de $G$, soit $N$ un sous-groupe normal de $G$. Rappelons la définition des bi-ensembles $\operatorname{Ind}_{H}^{G}, \operatorname{Inf}_{G / N}^{G}, \operatorname{Def}_{G / N}^{G}$ et $\operatorname{Res}_{H}^{G}$ : 
on a

$$
\begin{aligned}
\operatorname{Ind}_{H}^{G} & =(G \times H) /\{(g, g) \mid g \in H\} \\
\operatorname{Inf}_{G / N}^{G} & =(G \times(G / N)) /\{(g, g N) \mid g \in G\} \\
\operatorname{Def}_{G / N}^{G} & =((G / N) \times G) /\{(g N, g) \mid g \in G\} \\
\operatorname{Res}_{H}^{G} & =(H \times G) /\{(h, h) \mid h \in H\} .
\end{aligned}
$$

Soit $\varphi$ un isomorphisme d'un groupe fini $G$ dans un autre $G^{\prime}$, on définit le bi-ensemble $\operatorname{Iso}_{G^{\prime}}^{G}$ par

$$
\operatorname{Iso}_{G^{\prime}}^{G}=\left(G^{\prime} \times G\right) /\{(\varphi(g), g) \mid g \in G\} .
$$

La notation ne fait pas apparaître $\varphi$, mais cela ne créera aucune confusion en pratique.

Soient $G$ et $G^{\prime}$ deux groupes finis et $L$ un sous-groupe du produit $G \times G^{\prime}$, alors on note $p_{1}(L)$ (resp. $\left.p_{2}(L)\right)$ la projection de $L$ sur $G$ (resp. sur $G^{\prime}$ ).

De même on note

$$
k_{1}(L)=\{g \in G \mid(g, 1) \in L\} \text { et } k_{2}(L)=\left\{h \in G^{\prime} \mid(1, h) \in L\right\} .
$$

Pour tout $y$ élément de $p_{2}(L)$, il existe $x_{y}$ élément de $G$ tel que le couple $\left(x_{y}, y\right)$ soit élément de $L$. Alors en associant à $y k_{2}(L)$ l'élément $x_{y} k_{1}(L)$ on obtient un isomorphisme, dit canonique, entre $p_{2}(L) / k_{2}(L)$ et $p_{1}(L) / k_{1}(L)$.

Si $G^{\prime \prime}$ est un autre groupe fini, et $M$ un sous-groupe de $G^{\prime} \times G^{\prime \prime}$, alors on pose

$$
L * M=\left\{\left(g, g^{\prime \prime}\right) \in G \times G^{\prime \prime} \mid \exists g^{\prime} \in G^{\prime},\left(g, g^{\prime}\right) \in L,\left(g^{\prime}, g^{\prime \prime}\right) \in M\right\} .
$$

C'est un sous-groupe de $G \times G^{\prime \prime}$.

Rappelons la formule de Mackey relative aux bi-ensembles (cf. [1], 3.2): soient $G$ et $H$ deux groupes finis, soit $L$ un sous-groupe du produit $G \times H$ et $M$ un sous-groupe du produit $H \times K$. Alors

$$
(G \times H / L) \times_{H}(H \times K / M)=\sum_{h \in p_{2}(L) \backslash H / p_{1}(M)}(G \times K) /\left(L *^{(h, 1)} M\right) .
$$

Si $L$ est un sous-groupe du produit $G \times H$, alors d'après ([1], Lemme 3 , p. 672) le bi-ensemble $G \times H / L$ se décompose en un produit de morphismes dans $\mathcal{C}_{k}$ :

$$
G \times H / L=\operatorname{Ind}_{p_{1}(L)}^{G} \operatorname{Inf}_{p_{1}(L) / k_{1}(L)}^{p_{1}(L)} \operatorname{Iso}_{p_{1}(L) / k_{1}(L)}^{p_{2}(L) / k_{2}(L)} \operatorname{Def}_{p_{2}(L) / k_{2}(L)}^{p_{2}(L)} \operatorname{Res}_{p_{2}(L)}^{H}
$$

où $\operatorname{Iso}_{p_{1}(L) / k_{1}(L)}^{p_{2}(L) / k_{2}(L)}$ est relatif à l'isomorphisme canonique entre $p_{2}(L) / k_{2}(L)$ et $p_{1}(L) / k_{1}(L)$.

- Les foncteurs simples $S_{Q, k}$ : (cf. [1], Proposition 2).

Les objets simples de $\mathcal{F}_{k}$ sont paramétrés par des couples $(Q, V)$, où $Q$ est un $p$-groupe et $V$ est un $k \operatorname{Out}(Q)$-module simple. 
Si $S$ est un foncteur simple, le couple $(Q, V)$ correspondant est défini en prenant pour $Q$ un groupe d'ordre minimal tel que $S(Q)$ est différent de 0 et en considérant $V=S(Q)$ comme $k \operatorname{Out}(Q)$-module.

Inversement, étant donné un couple $(Q, V)$ on a un foncteur simple noté $S_{Q, V}$ défini, pour un $p$-groupe $P$, par

$$
\begin{array}{r}
S_{Q, V}(P)=\left(\operatorname{Hom}_{\mathcal{C}_{k}}(Q, P) \otimes_{\operatorname{End}_{\mathcal{C}_{k}}(Q)} V\right) /\left\{\sum_{i} \varphi_{i} \otimes v_{i} \mid\right. \\
\left.\forall \psi \in \operatorname{Hom}_{\mathcal{C}_{k}}(P, Q), \sum_{i}\left(\psi \varphi_{i}\right) v_{i}=0\right\} .
\end{array}
$$

La notation $S_{Q, k}$ désignera le foncteur simple associé au $k$ Out $(Q)$-module trivial $k$.

\section{Certains foncteurs simples en caractéristique $p$}

Lemme 1. Soient $Q$ et $P$ deux p-groupes finis, soit $A$ un sous-End $(Q)$ module de $\mathcal{B}(Q)$ et $J$ un sous-End $(Q)$-module maximal de $A$.

Soit $\mathcal{N}_{Q, A}$ le sous-foncteur du foncteur de Burnside B, défini par

$$
\mathcal{N}_{Q, A}(P)=\operatorname{Hom}_{\mathcal{C}_{k}}(Q, P) \times_{Q} A
$$

et $\mathcal{N}_{Q, A, J}$ le sous-foncteur de $\mathcal{N}_{Q, A}$, défini par

$$
\mathcal{N}_{Q, A, J}(P)=\left\{u \in \mathcal{N}_{Q, A}(P) \mid \forall \varphi \in \operatorname{Hom}_{\mathcal{C}_{k}}(P, Q):\left(\varphi \times{ }_{P} u\right) \in J\right\} .
$$

La correspondance qui à $J$ associe $\mathcal{N}_{Q, A, J}$, est une bijection entre l'ensemble des sous-End $(Q)$-modules maximaux de $A$ et l'ensemble des sous-foncteurs maximaux de $\mathcal{N}_{Q, A}$.

Preuve. Vérifions que $\mathcal{N}_{Q, A, J}$ est un sous-foncteur de $\mathcal{N}_{Q, A}$ :

Soient $P$ et $P^{\prime}$ deux $p$-groupes finis, soit $\psi \in \operatorname{Hom}_{\mathcal{C}_{k}}\left(P^{\prime}, P\right)$, vérifions qu'on a

$$
\psi \times{ }_{P^{\prime}} N_{Q, A, J}\left(P^{\prime}\right) \subset N_{Q, A, J}(P) .
$$

D'après l'associativité du produit des bi-ensembles, on a

$$
\forall u \in N_{Q, A, J}\left(P^{\prime}\right), \forall \psi^{\prime} \in \operatorname{Hom}_{\mathcal{C}_{k}}(P, Q), \psi^{\prime} \times_{P}\left(\psi \times_{P^{\prime}} u\right)=\left(\psi^{\prime} \times_{P} \psi\right) \times_{P^{\prime}} u .
$$

Or d'après la définition de $N_{Q, A, J}\left(P^{\prime}\right)$ :

$$
\operatorname{Hom}_{\mathcal{C}_{k}}\left(P^{\prime}, Q\right) \times P_{P^{\prime}} u \subset J
$$

et $\left(\psi^{\prime} \times_{P} \psi\right) \in \operatorname{Hom}_{\mathcal{C}_{k}}\left(P^{\prime}, Q\right)$, donc $\left(\psi^{\prime} \times_{P} \psi\right) \times{ }_{P^{\prime}} u \in J$, ainsi $\psi^{\prime} \times{ }_{P}\left(\psi \times{ }_{P^{\prime}} u\right)$ est dans $J$, et $N_{Q, A, J}$ est un sous-foncteur de $N_{Q, A}$. De plus $\mathcal{N}_{Q, A, J} \neq \mathcal{N}_{Q, A}$, car par exemple $\mathcal{N}_{Q, A, J}(Q)=J$ alors que $\mathcal{N}_{Q, A}(Q)=A$.

Soit $L$ un sous-foncteur de $\mathcal{N}_{Q, A}$, en particulier on a $L(Q) \subset \mathcal{N}_{Q, A}(Q)=$ $A$. Alors il y a deux cas à envisager. 
(1) Ou bien $L(Q) \subset J$, dans ce cas

$$
\forall u \in L(P), \forall \varphi \in \operatorname{Hom}_{\mathcal{C}_{k}}(P, Q):\left(\varphi \times{ }_{P} u\right) \in L(Q)
$$

donc $L \subset \mathcal{N}_{Q, A, J}$.

(2) Ou bien $L(Q) \not \subset J$, donc $L(Q)+J=A$ et par suite $L+\mathcal{N}_{Q, J}=\mathcal{N}_{Q, A}$, car

$$
\begin{aligned}
N_{Q, A}(P) & =\operatorname{Hom}_{\mathcal{C}_{k}}(Q, P) \times_{Q} A \\
& =\operatorname{Hom}_{\mathcal{C}_{k}}(Q, P) \times_{Q}(L(Q)+J) \\
& \left.=\operatorname{Hom}_{\mathcal{C}_{k}}(Q, P) \times_{Q} L(Q)\right)+N_{Q, J}(P) .
\end{aligned}
$$

Comme $L$ est un sous-foncteur de $N_{Q, A}$, on a

$$
\operatorname{Hom}_{\mathcal{C}_{k}}(Q, P) \times{ }_{Q} L(Q) \subset L(P)
$$

d'où

$$
N_{Q, A}(P) \subset L(P)+N_{Q, J}(P) .
$$

On a alors

$$
L+\mathcal{N}_{Q, J}=\mathcal{N}_{Q, A}
$$

or $\mathcal{N}_{Q, J} \subset \mathcal{N}_{Q, A, J}$, alors

$$
L+\mathcal{N}_{Q, A, J}=\mathcal{N}_{Q, A} .
$$

D'après ce qui précède on a établi que si $L$ est sous-foncteur de $\mathcal{N}_{Q, A}$ alors ou bien $L \subset \mathcal{N}_{Q, A, J}$ ou bien $L+\mathcal{N}_{Q, A, J}=\mathcal{N}_{Q, A}$, donc $\mathcal{N}_{Q, A, J}$ est un sousfoncteur maximal de $\mathcal{N}_{Q, A}$, et le quotient $\mathcal{N}_{Q, A} / \mathcal{N}_{Q, A, J}$ est un foncteur simple.

D'autre part, si $L$ est un sous-foncteur maximal (propre) de $\mathcal{N}_{Q, A}$, alors $L(Q) \neq A$, car sinon on aurait $\operatorname{Hom}_{\mathcal{C}_{k}}(Q, P) \times{ }_{Q} L(Q)=\operatorname{Hom}_{\mathcal{C}_{k}}(Q, P) \times_{Q} A$, i.e., $\mathcal{N}_{Q, A}=L$. Il existe un sous-End $(Q)$-module maximal de $A$, noté $J_{L}$ tel que $L(Q) \subset J_{L}$, par le premier cas on déduit que $L \subset \mathcal{N}_{Q, A, J_{L}}$, et comme $L$ est un sous-foncteur maximal de $\mathcal{N}_{Q, A}$ on a $L=\mathcal{N}_{Q, A, J_{L}}$.

Finalement, si $J$ et $J^{\prime}$ sont deux sous-End $(Q)$-modules maximaux de $A$ tels que $J \neq J^{\prime}$, alors $\mathcal{N}_{Q, A, J} \neq \mathcal{N}_{Q, A, J^{\prime}}$, car par exemple $\mathcal{N}_{Q, A, J}(Q)=J$ alors que $\mathcal{N}_{Q, A, J^{\prime}}(Q)=J^{\prime}$.

Soient $H$ et $P$ deux $p$-groupes finis, dans toute la suite on notera " $H$ sous-groupe de $P$ " par $H \prec P$.

Soit $Q$ un $p$-groupe fini non-trivial. Considérons dans $k \otimes_{\mathbb{Z}} B(Q)$ le $Q$ ensemble virtuel $\xi_{Q}$, défini par

$$
\xi_{Q}=Q / 1-\sum_{\substack{|Z|=p \\ Z \prec \mathcal{Z}(Q)}} Q / Z
$$

où $\mathcal{Z}(Q)$ désigne le centre du groupe $Q$.

Lemme 2. Soit $Q$ un p-groupe non-trivial, alors dans $k \otimes_{\mathbb{Z}} B$ on a: 
- $\operatorname{Def}_{Q / N}^{Q} \xi_{Q}=0$, si $N \unlhd Q$ et $N \neq 1$.

- $\operatorname{Res}_{P}^{Q} \xi_{Q}=0$, si $Q$ est d'ordre au moins $p^{2}$ et $P$ est un sous-groupe propre de $Q$.

Preuve.

- Soit $N \unlhd Q$ et $N \neq 1$. Rappelons que si $H$ est un sous-groupe de $Q$, alors

$$
\operatorname{Def}_{Q / N}^{Q} Q / H=(Q / N) /(H \cdot N / N) .
$$

Ainsi

$$
\begin{aligned}
\operatorname{Def}_{Q / N}^{Q} \xi_{Q}= & (Q / N) /(N / N)-\sum_{\substack{|Z|=p \\
Z \prec \mathcal{Z}(Q)}}(Q / N) /(Z \cdot N / N) \\
= & (Q / N) /(N / N)-\sum_{\substack{|Z \prec|=p \\
Z \prec \mathcal{Z}(Q) \cap N}}(Q / N) /(N / N) \\
& -\sum_{\substack{|Z|=p \\
Z \prec \mathcal{Z}(Q) \\
Z \not \subset N}}(Q / N) /(Z \cdot N / N) .
\end{aligned}
$$

Or $N$ est un sous-groupe normal non-trivial de $Q$, donc $N \cap \mathcal{Z}(Q) \neq$ 1 , et $|\{Z \prec(\mathcal{Z}(Q) \cap N),|Z|=p\}|$ est le nombre de sous-groupes d'ordre $p$ qui engendrent le $p$-groupe abélien élémentaire non-trivial $\Omega_{1}(\mathcal{Z}(Q) \cap N)$, donc

$$
|\{Z \prec(\mathcal{Z}(Q) \cap N),|Z|=p\}|=\frac{\left|\Omega_{1}(\mathcal{Z}(Q) \cap N)\right|-1}{p-1}
$$

par suite

$$
|\{Z \prec(\mathcal{Z}(Q) \cap N),|Z|=p\}| \equiv 1(p) .
$$

Donc

$$
\operatorname{Def}_{Q / N}^{Q} \xi_{Q}=-\sum_{\substack{|Z|=p \\ Z \prec \mathcal{Z}(Q) \\ Z \not \subset N}}(Q / N) /(Z \cdot N / N)
$$

ou encore

$$
\operatorname{Def}_{Q / N}^{Q} \xi_{Q}=-\sum_{\substack{M \prec Q \\|M: N|=p}} \sum_{\substack{\mid Z \prec \mathcal{Z}(Q) \\ Z \not N N \\ Z \cdot N=M}}(Q / N) /(M / N) .
$$

Or si $|M: N|=p$, alors

$\mid\{Z \prec \mathcal{Z}(Q)$ tels que $Z \not \subset N, Z \cdot N=M$ et $|Z|=p\} \mid=n_{1}-n_{2}$ 
où

$$
\begin{aligned}
& n_{1}=\mid\{Z \prec \mathcal{Z}(Q) \cap M \text { tels que }|Z|=p\} \mid \\
& n_{2}=\mid\{Z \prec \mathcal{Z}(Q) \cap N \cap M \text { tels que }|Z|=p\} \mid
\end{aligned}
$$

avec $n_{1} \equiv 1(p)$ et $n_{2} \equiv 1(p)$, donc

$$
\mid\{Z \prec \mathcal{Z}(Q) \text { tels que } Z \not \subset N, Z \cdot N=M \text { et }|Z|=p\} \mid \equiv 0(p)
$$

d'où $\operatorname{Def}_{Q / N}^{Q} \xi_{Q}=0$.

- D'après la formule de Mackey on a

$$
\begin{aligned}
\operatorname{Res}_{P}^{Q} \xi_{Q} & =\sum_{x \in(P \backslash Q / 1)} P /\left(P \cap^{x} 1\right)-\sum_{\substack{|Z|=p \\
Z \prec \mathcal{Z}(Q)}} \sum_{x \in(P \backslash Q / Z)} P /\left(P \cap \cap^{x} Z\right) \\
= & |Q: P| \cdot P / 1-\sum_{\substack{|Z|=p \\
Z \prec \mathcal{Z}(Q)}}|Q: P \cdot Z| \cdot P /(P \cap Z) .
\end{aligned}
$$

Pour cela on distinguera deux cas:

(1) Si $|Q: P| \geq p^{2}$, alors

$$
\forall Z \prec \mathcal{Z}(Q),|Z| \leq p \text {, on a }|Q: P \cdot Z| \geq p \text {. }
$$

Donc $\operatorname{Res}_{P}^{Q} \xi_{Q}=0$.

(2) Si $|Q: P|=p$, alors

$$
\begin{aligned}
\operatorname{Res}_{P}^{Q} \xi_{Q} & =-\sum_{\substack{|Z|=p \\
Z \prec \mathcal{Z}(Q) \\
Z \not \subset P}} P /(P \cap Z) \\
& =-\mid\{Z \prec \mathcal{Z}(Q) \text { tels que } Z \not \subset P \text { et }|Z|=p\} \mid \cdot P / 1 .
\end{aligned}
$$

Comme $|Q: P|=p$ et $|Q| \geq p^{2}$, alors le sous-groupe $P$ est nontrivial. Comme de plus $P$ est normal dans $Q$, alors $P \cap \mathcal{Z}(Q) \neq 1$, et par suite

$$
\mid\{Z \prec \mathcal{Z}(Q) \text { tels que } Z \not \subset P \text { et }|Z|=p\} \mid \equiv 0(p) \text {. }
$$

D'où $\operatorname{Res}_{P}^{Q} \xi_{Q}=0$.

Lemme 3. Dans $k \otimes_{\mathbb{Z}} B(Q)$, on a $\operatorname{End}(Q) \times_{Q} \xi_{Q}$ est isomorphe à $k$. C'est un $k$ Out $(Q)$-module trivial.

Preuve. Pour tout sous-groupe $L$ de $Q \times Q$, on a:

$$
(Q \times Q) / L=\operatorname{Ind}_{p_{1}(L)}^{Q} \operatorname{Inf}_{p_{1}(L) / k_{1}(L)}^{p_{1}(L)} \operatorname{Iso}_{p_{1}(L) / k_{1}(L)}^{p_{2}(L) / k_{2}(L)} \operatorname{Def}_{p_{2}(L) / k_{2}(L)}^{p_{2}(L)} \operatorname{Res}_{p_{2}(L)}^{Q}
$$
et

$$
L=\{(g, h) \in Q \times Q \mid s(g)=t(h)\}
$$


où $s$ est une surjection d'un sous-groupe $P_{1}$ de $Q$ dans un groupe $Q^{\prime}$, et $t$ une surjection d'un sous-groupe $P_{2}$ de $Q$ dans $Q^{\prime}$.

D'après le Lemme 2, on a

$$
(Q \times Q / L) \times{ }_{Q} \xi_{Q} \neq 0 \Rightarrow p_{2}(L)=Q \text { et } k_{2}(L)=1 .
$$

Il en résulte que $Q^{\prime} \simeq Q$.

Or $Q^{\prime} \simeq p_{1}(L) / k_{1}(L) \simeq p_{2}(L) / k_{2}(L)$, donc $p_{1}(L)=Q$ et $k_{1}(L)=1$, par suite $s$ et $t$ sont des isomorphismes.

Ainsi $L$ devient

$$
L=\triangle_{s^{-1} \circ t}(Q)=\triangle_{\varphi}(Q)
$$

où

$$
\varphi=s^{-1} \circ t \in \operatorname{Aut}(Q)
$$

Dans ce cas

$$
\left(Q \times Q / \triangle_{\varphi}(Q)\right) \times_{Q} \xi_{Q}=Q / \varphi(1)-\sum_{\substack{|Z|=p \\ Z \prec \mathcal{Z}(Q)}} Q / \varphi(Z) .
$$

Donc

$$
\begin{aligned}
\left(Q \times Q / \triangle_{\varphi}(Q)\right) \times_{Q} \xi_{Q} & =Q / 1-\sum_{\substack{|Z|=p \\
Z \prec \mathcal{Z}(Q)}} Q / Z \\
& =\xi_{Q}
\end{aligned}
$$

et par suite

$$
\operatorname{End}(Q) \times_{Q} \xi_{Q}=k \xi_{Q}
$$

D'autre part, on a

$$
\operatorname{End}(Q)=k \operatorname{Out}(Q) \oplus I_{Q} \quad(\text { cf. [1], Corollaire, p. 677) }
$$

où $I_{Q}$ est l'idéal de $\operatorname{End}(Q)$ engendré par des bi-ensembles factorisant par des groupes $K$ d'ordre strictement inférieur à celui de $Q$.

On a $I_{Q} \times_{Q} \xi_{Q}=0$, car les générateurs de $I_{Q}$ sont de la forme

$$
(Q \times K) / L \times_{K}(K \times Q) / M .
$$

Puisque $|K|<|Q|$, alors par le Lemme 2 on a $[(K \times Q) / M] \times{ }_{Q} \xi_{Q}=0$ :

En effet

$$
\left|p_{2}(M)\right| /\left|k_{2}(M)\right|=\left|p_{1}(M)\right| /\left|k_{1}(M)\right| \leq|K|<|Q|
$$

donc

il s'ensuit que

$$
\operatorname{Def}_{p_{2}(M) / k_{2}(M)}^{p_{2}(M)} \operatorname{Res}_{p_{2}(M)}^{Q} \xi_{Q}=0
$$

$$
[(K \times Q) / M] \times{ }_{Q} \xi_{Q}=0
$$

et par conséquent

$$
\left[(Q \times K) / L \times_{K}(K \times Q) / M\right] \times_{Q} \xi_{Q}=0 .
$$


Ainsi $I_{Q} \times{ }_{Q} \xi_{Q}=0$ et $\operatorname{End}(Q) \times{ }_{Q} \xi_{Q}$ est un $k \operatorname{Out}(Q)$-module trivial.

Proposition 1. Soit $Q$ un p-groupe d'ordre au moins $p^{2}$. Considérons le sous-foncteur $F_{Q}$ de $k \otimes_{\mathbb{Z}} B$ défini, pour un p-groupe fini $P$, par:

$$
F_{Q}(P)=\operatorname{Hom}_{\mathcal{C}_{k}}(Q, P) \times{ }_{Q} \xi_{Q}
$$

et le sous-foncteur $J_{Q}$ de $F_{Q}$ défini, pour un p-groupe fini $P$, par:

$$
J_{Q}(P)=\left\{u \in F_{Q}(P) \mid \forall \varphi \in \operatorname{Hom}_{\mathcal{C}_{k}}(P, Q), \varphi \times_{P} u=0\right\} .
$$

Alors $J_{Q}$ est l'unique sous-foncteur maximal de $F_{Q}$, et on a $F_{Q} / J_{Q} \simeq S_{Q, k}$.

Puis considérons le sous-foncteur $J_{1, k}$ de $k \otimes_{\mathbb{Z}} B$ défini, pour un p-groupe fini $P$, par:

$$
J_{1, k}(P)=\left\{u \in k \otimes_{\mathbb{Z}} B(P) \mid \forall \varphi \in \operatorname{Hom}_{\mathcal{C}_{k}}(P, 1), \varphi \times_{P} u=0\right\}
$$

i.e.,

$$
J_{1, k}(P)=\left\{X \in k \otimes_{\mathbb{Z}} B(P) \mid \forall U \text { sous-groupe de } P,|U \backslash X|=0\right\} .
$$

Alors $J_{1, k}$ est l'unique sous-foncteur maximal de $k \otimes_{\mathbb{Z}} B$, et on a $\left(k \otimes_{\mathbb{Z}}\right.$ B) $/ J_{1, k} \simeq S_{1, k}$.

Preuve. L'existence et l'unicité du foncteur $J_{Q}$ dans $F_{Q}$ résultent du Lemme 1 et du Lemme 3 . On sait aussi que $F_{Q} / J_{Q}$ est simple, il reste à vérifier que $F_{Q} / J_{Q} \simeq S_{Q, k}$ :

On a $F_{Q}(Q) / J_{Q}(Q)=\operatorname{End}(Q) \times{ }_{Q} \xi_{Q}$ qui est un $k \operatorname{Out}(Q)$-module trivial [cf. Lemme 3]. De plus

$$
\forall K p \text { - groupe tel que }|K|<|Q|, F_{Q}(K) / J_{Q}(K)=0 .
$$

En effet, pour tout sous-groupe $L$ de $K \times Q$, on a $((K \times Q) / L) \times{ }_{Q} \xi_{Q}=0$.

Donc

$$
F_{Q}(K)=0 \text {, si }|K|<|Q| \text {. }
$$

D'autre part, on a

$$
k \otimes_{\mathbb{Z}} B(P)=\operatorname{Hom}_{\mathcal{C}_{k}}(1, P) \times_{1} \operatorname{End}(1) .
$$

Sachant que End(1) est réduit à $k$, alors par le Lemme $1 k \otimes_{\mathbb{Z}} B$ a un unique sous-foncteur maximal $J_{1, k}$ défini, pour un $p$-groupe fini $P$, par:

$$
J_{1, k}(P)=\left\{u \in k \otimes_{\mathbb{Z}} B(P) \mid \forall \varphi \in \operatorname{Hom}_{\mathcal{C}_{k}}(P, 1), \varphi \times_{P} u=0\right\} .
$$

De plus $\left(k \otimes_{\mathbb{Z}} B\right)(1) / J_{1, k}(1)$ est le $k \operatorname{Out}(Q)$-module trivial $k$. D'où $\left(k \otimes_{\mathbb{Z}}\right.$ $B) / J_{1, k} \simeq S_{1, k}$.

Finalement, pour tout sous-groupe $U$ de $P$, si $X$ est un élément de $k \otimes_{\mathbb{Z}}$ $B(P)$, alors

$$
((1 \times P) /(1 \times U)) \times{ }_{P} X=|U \backslash X| .
$$

Par suite

$$
J_{1, k}(P)=\left\{X \in k \otimes_{\mathbb{Z}} B(P) \mid \forall U \text { sous-groupe de } P,|U \backslash X|=0\right\} .
$$

D'où l'équivalence entre les deux formules de $J_{1, k}(P)$. 
2.1. Le cas des foncteurs simples $S_{Q, k}$, avec $|Q| \geq p^{2}$. Soit $K$ un sous-groupe d'un groupe fini $P$, et $N$ un sous-groupe normal de $K$, on dit que $(K, N)$ est une section de $P$. Soit $H$ un autre groupe, on note $\Sigma_{H}(P)$ l'ensemble des sections $(K, N)$ de $P$ telles que $K / N \simeq H$.

Rappelons la définition de deux sections liées (cf. [1], p. 685):

Soient $K \supset N$ et $L \supset M$ des sous-groupes de $P$, les sections $(K, N)$ et $(L, M)$ sont liées, si

$$
(K \cap L) \cdot N=K,(K \cap L) \cdot M=L, K \cap M=L \cap N .
$$

On note $B_{Q}(P)$ l'espace vectoriel sur $k$ ayant pour base les classes de conjugaison par $P$ de sections $(K, N)$, éléments de $\Sigma_{Q}(P)$. D'après $([\mathbf{1}]$, p. 717), $\operatorname{dim}_{k} S_{Q, k}(P)$ est égale au rang de la forme bilinéaire sur $B_{Q}(P)$ à valeurs dans $k$ définie par:

$$
\langle(K, N),(L, M)\rangle_{P}=\mid\left\{x \in(K \backslash P / L) \mid(K, N) \text { et }\left({ }^{x} L,{ }^{x} M\right) \text { sont liées }\right\} \mid .
$$

Proposition 2. Soient $Q$ et $P$ deux p-groupes finis tel que l'ordre de $Q$ est au moins $p^{2}$, alors

$$
F_{Q}(P)=\left\langle P / R-\sum_{\substack{|Z / R|=p \\ Z / R \prec \mathcal{Z}(S / R)}} P / Z \mid(S, R) \in \Sigma_{Q}(P)\right\rangle
$$

où $F_{Q}$ est le sous-foncteur défini dans Proposition 1.

Preuve. Soit $(S, R)$ un élément de $\Sigma_{Q}(P)$, alors

$$
\left(P / R-\sum_{\substack{|Z / R|=p \\ Z / R \prec \mathcal{Z}(S / R)}} P / Z\right)=\left(\operatorname{Ind}_{S}^{P} \operatorname{Inf}_{S / R}^{S} \xi_{S / R}\right) \in F_{Q}(P)
$$

donc

$$
\left\langle P / R-\sum_{\substack{|Z / R|=p \\ Z / R \prec \mathcal{Z}(S / R)}} P / Z \mid(S, R) \in \Sigma_{Q}(P)\right\rangle \subseteq F_{Q}(P) .
$$

Inversement, un générateur de $F_{Q}(P)$ est de la forme $(P \times Q) / L \times_{Q} \xi_{Q}$, où $L$ est un sous-groupe du produit $P \times Q$, alors en utilisant la décomposition de $(P \times Q) / L$ puis en appliquant le Lemme 2 à $(P \times Q) / L \times{ }_{Q} \xi_{Q}$, on obtient

$$
(P \times Q) / L \times_{Q} \xi_{Q}=\operatorname{Ind}_{S}^{P} \operatorname{Inf}_{S / R}^{S} \operatorname{Iso}_{S / R}^{Q} \xi_{Q}=P / R-\sum_{\substack{|Z / R|=p \\ Z / R \prec \mathcal{Z}(S / R)}} P / Z
$$

pour une section $(S, R)$ convenable, d'où la proposition. 
Lemme 4. Soit $P$ un p-groupe d'ordre au moins $p^{2}$, et $M_{0}$ un sous-groupe maximal de $P$, alors

$$
F_{M_{0}}(P)=J_{M_{0}}(P) \oplus\left\langle P / Z-\sum_{\substack{|E / Z|=p \\ E / Z \prec \mathcal{Z}(P / Z)}} P / E \mid(P, Z) \in \Sigma_{M_{0}}(P)\right\rangle
$$

et

$$
\operatorname{dim}_{k} S_{M_{0}, k}(P)=\left|\left\{Z \prec \mathcal{Z}(P) \mid(P / Z) \cong M_{0}\right\}\right| .
$$

Preuve. Calculons $\operatorname{dim}_{k} S_{M_{0}, k}(P)$ :

On a $\operatorname{dim}_{k} S_{M_{0}, k}(P)$ est égale au rang de la matrice suivante

$$
\mathcal{I}=\left(\langle(K, N),(L, M)\rangle_{P}\right)_{(K, N),(L, M) \in B_{M_{0}}(P)}
$$

ou encore

$$
\mathcal{I}=\left(\begin{array}{cc}
I_{r} & A \\
{ }^{t} A & O
\end{array}\right)
$$

On désigne $\operatorname{par}{ }^{t} A$ la matrice transposée de $A$, qui est obtenue par la restriction de la forme bilinéaire de $B_{M_{0}}(P)$ aux sections $[(P, U),(M, 1)]$, alors que la matrice $I_{r}$ est obtenue par la restriction aux sections $[(P, U),(P, V)]$. Il en résulte que $I_{r}$ est la matrice unité de rang $r$, où $r=\mid\{Z \prec \mathcal{Z}(P) \mid(P / Z) \simeq$ $\left.M_{0}\right\}$. La matrice $O$ est quant à elle égale à la matrice nulle de rang le nombre de sous-groupes maximaux de $P$ isomorphes à $M_{0}$, car

$$
O=\left(\left\langle(M, 1),\left(M^{\prime}, 1\right)\right\rangle_{P}\right)_{(M, 1),\left(M^{\prime}, 1\right) \in B_{M_{0}}(P)} .
$$

On remarque que les coefficients de la matrice ${ }^{t} A \cdot A$ sont de la forme

$$
\lambda_{M, M^{\prime}}=\sum_{\substack{(P, Z) \in \Sigma_{M_{0}}(P) \\ Z \not \subset M \\ Z \not \subset M^{\prime}}} 1
$$

où la section $(M, 1) \in \Sigma_{M_{0}}(P)$. Donc

$$
\lambda_{M, M^{\prime}}=\sum_{\substack{Z \prec \Omega_{1}(\mathcal{Z}(P)) \\ Z \not \subset M \\ Z \not \subset M^{\prime}}} 1 .
$$

Or

$$
\begin{aligned}
\sum_{\substack{Z \prec \Omega_{1}(\mathcal{Z}(P)) \\
Z \not \subset M}} 1 & =\sum_{\substack{Z \prec \Omega_{1}(\mathcal{Z}(P))\\
}} 1-\sum_{\substack{Z \prec \Omega_{1}(\mathcal{Z}(P)) \\
Z \subset M}} 1 \\
& \equiv 0(p)
\end{aligned}
$$


alors certainement

$$
\begin{aligned}
\sum_{\substack{Z \prec \Omega_{1}(\mathcal{Z}(P)) \\
Z \not \subset M \\
Z \not \subset M^{\prime}}} 1 & =\sum_{\substack{Z \prec \Omega_{1}(\mathcal{Z}(P)) \\
Z \not \subset M}} 1-\sum_{\substack{Z \prec \Omega_{1}(\mathcal{Z}(P)) \\
Z \not \subset M \\
Z \subset M^{\prime}}} 1 \\
& \equiv-\sum_{\substack{Z \prec \Omega_{1}(\mathcal{Z}(P)) \\
Z \not \subset M \\
Z \subset M^{\prime}}} 1 \\
& \equiv 0(p) .
\end{aligned}
$$

Ainsi $\lambda_{M, M^{\prime}} \equiv 0(p)$, et on trouve ${ }^{t} A \cdot A=0$.

Montrons ensuite que

$$
\operatorname{rg} \mathcal{I}=r+\operatorname{rg}\left({ }^{t} A \cdot A\right) .
$$

Associons respectivement aux matrices $\mathcal{I}$ et ${ }^{t} A \cdot A$, les applications $k$-linéaires $f$ et $g$. Considérons le vecteur $T$

$$
T=\left(\begin{array}{c}
X \\
Y
\end{array}\right) \in \operatorname{Ker}(\mathrm{f}) .
$$

Donc

$$
\mathcal{I} \cdot T=\mathcal{I}\left(\begin{array}{c}
X \\
Y
\end{array}\right)=0
$$

On obtient le système suivant

$$
\left\{\begin{array}{c}
X+A Y=0 \\
{ }^{t} A X=0
\end{array}\right.
$$

qui est équivalent au système

$$
\left\{\begin{array}{c}
X=-A Y \\
\left({ }^{t} A \cdot A\right) Y=0
\end{array}\right.
$$

Ainsi

$$
\operatorname{dim}_{k} \operatorname{Ker}(\mathrm{f})=\operatorname{dim}_{k} \operatorname{Ker}(\mathrm{g})
$$

par suite

$$
\operatorname{rg} \mathcal{I}=r+\operatorname{rg}\left({ }^{t} A \cdot A\right)=r .
$$

D'où

$$
\operatorname{dim}_{k} S_{M_{0}, k}(P)=\left|\left\{Z \prec \mathcal{Z}(P) \mid(P / Z) \cong M_{0}\right\}\right| .
$$

D'autre part, on a:

$$
F_{M_{0}}(P)=J_{M_{0}}(P) \oplus\left\langle P / Z-\sum_{\substack{|E / Z|=p \\ E / Z \prec \mathcal{Z}(P / Z)}} P / E \mid(P, Z) \in \Sigma_{M_{0}}(P)\right\rangle .
$$


En effet: soit

$$
x=\sum_{(P, Z) \in \Sigma_{M_{0}}(P)} \lambda_{Z}\left(P / Z-\sum_{\substack{|E / Z|=p \\ E / Z \prec \mathcal{Z}(P / Z)}} P / E\right) \in J_{M_{0}}(P) .
$$

Fixons une section $\left(P, Z_{0}\right) \in \Sigma_{M_{0}}(P)$ et appliquons $\operatorname{Def}_{P / Z_{0}}^{P}$ à $x$. On a donc $\operatorname{Def}_{P / Z_{0}}^{P}(x)=0$, dans $k \otimes_{\mathbb{Z}} B\left(P / Z_{0}\right)$

i.e.,

$$
\begin{aligned}
& 0=\lambda_{Z_{0}}\left[\left(P / Z_{0}\right) /\left(Z_{0} / Z_{0}\right)-\sum_{\substack{\left|E / Z_{0}\right|=p \\
E / Z_{0} \prec \mathcal{Z}\left(P / Z_{0}\right)}}\left(P / Z_{0}\right) /\left(E \cdot Z_{0} / Z_{0}\right)\right] \\
& +\sum_{\substack{\left(P, Z^{\prime}\right) \in \Sigma_{M_{0}}(P) \\
Z^{\prime} \neq Z_{0}}} \lambda_{Z^{\prime}}\left[\left(P / Z_{0}\right) /\left(Z^{\prime} \cdot Z_{0} / Z_{0}\right)\right. \\
& \left.-\sum_{\substack{\left|E^{\prime} / Z^{\prime}\right|=p \\
E^{\prime} / Z^{\prime} \prec \mathcal{Z}\left(P / Z^{\prime}\right)}}\left(P / Z_{0}\right) /\left(E^{\prime} \cdot Z_{0} / Z_{0}\right)\right] .
\end{aligned}
$$

Dans cette égalité le terme $\left(P / Z_{0}\right) /\left(Z_{0} / Z_{0}\right)$ est unique, donc $\lambda_{Z_{0}}=0$. Comme la section $\left(P, Z_{0}\right)$ élément de $\Sigma_{M_{0}}(P)$, est arbitraire, on conclut que $x=0$. Par suite, dans $F_{M_{0}}(P)$ on a

$$
J_{M_{0}}(P) \cap\left\langle P / Z-\sum_{\substack{|E / Z|=p \\ E / Z \prec \mathcal{Z}(P / Z)}} P / E \mid(P, Z) \in \Sigma_{M_{0}}(P)\right\rangle=\{0\} .
$$

Or

$$
\begin{aligned}
\operatorname{dim}_{k} S_{M_{0}, k}(P) & =\operatorname{dim}_{k}\left(F_{M_{0}}(P) / J_{M_{0}}(P)\right) \\
& =\left|\left\{Z \prec \mathcal{Z}(P) \mid(P / Z) \cong M_{0}\right\}\right|
\end{aligned}
$$

ce qui prouve que

$$
F_{M_{0}}(P)=J_{M_{0}}(P) \oplus\left\langle P / Z-\sum_{\substack{|E / Z|=p \\ E / Z \prec \mathcal{Z}(P / Z)}} P / E \mid(P, Z) \in \Sigma_{M_{0}}(P)\right\rangle .
$$


Proposition 3. Soient $P$ et $Q$ deux p-groupes finis, tel que l'ordre de $Q$ est au moins $p^{2}$, alors

$$
\begin{gathered}
F_{Q}(P)=J_{Q}(P)+\left\langle P / R-\sum_{\substack{|Z / R|=p \\
Z / R \prec \mathcal{Z}(Q)}} P / Z \mid\left(N_{P}(R) / R\right) \cong Q\right\rangle \\
\supseteq J_{Q}(P) \oplus\left\langle P / R-\sum_{\substack{|Z / R|=p \\
Z / R \prec \mathcal{Z}(Q)}} P / Z \mid(P, R) \in \Sigma_{Q}(P)\right\rangle
\end{gathered}
$$

et on a

$$
\begin{aligned}
& \mid\{\text { sous-groupes normaux } R \text { de } P \mid(P / R) \cong Q\} \mid \\
& \leq \operatorname{dim}_{k} S_{Q, k}(P) \\
& \leq \mid\left\{\text { classes de conjugaison de sous-groupes } R \text { de } P \mid\left(N_{P}(R) / R\right) \cong Q\right\} \mid .
\end{aligned}
$$

Preuve. Soit $\left(S_{0}, R_{0}\right)$ un élément de $\Sigma_{Q}(P)$, montrons par récurrence sur l'indice $\left[P: S_{0}\right]$, que le couple $\left(S_{0}, R_{0}\right)$ vérifie la propriété $\mathcal{P}$ suivante:

$$
\begin{aligned}
& \left(P / R_{0}-\sum_{\substack{\left|Z / R_{0}\right|=p \\
Z / R_{0} \prec \mathcal{Z}\left(S_{0} / R_{0}\right)}} P / Z\right) \in \\
& J_{Q}(P)+\left\langle P / R-\sum_{\substack{|Z / R|=p \\
Z / R \prec \mathcal{Z}(Q)}} P / Z \mid\left(N_{P}(R) / R\right) \cong Q\right\rangle .
\end{aligned}
$$

Si $\left|P / S_{0}\right|=1$, alors $R_{0}$ est normal dans $P$, par suite la propriété $\mathcal{P}$ est vérifiée.

Soit $\left(S_{0}, R_{0}\right)$ un élément de $\Sigma_{Q}(P)$ tel que $\left|P / S_{0}\right|=p^{n+1}$. Supposons que la propriété $\mathcal{P}$ est vérifiée pour les sections $(S, R) \in \Sigma_{Q}(P)$ telles que $|P / S|=p^{n}$, et montrons que la section $\left(S_{0}, R_{0}\right)$ vérifie aussi la propriété $\mathcal{P}$ :

Si $S_{0}=N_{P}\left(R_{0}\right)$ alors la propriété $\mathcal{P}$ est vérifiée. Si $S_{0}$ est un sous-groupe propre de $N_{P}\left(R_{0}\right)$, il existe un sous-groupe $T$ de $P$ tel que $T$ contient $S_{0}$, le sous-groupe $R_{0}$ est normal dans $T$ et $\left|T / S_{0}\right|=p$, alors par la Proposition 2 on a

$$
y=\left(T / R_{0}\right) /\left(R_{0} / R_{0}\right)-\sum_{\substack{\left|Z / R_{0}\right|=p \\ Z / R_{0} \prec \mathcal{Z}\left(S_{0} / R_{0}\right)}}\left(T / R_{0}\right) /\left(Z / R_{0}\right) \in F_{Q}\left(T / R_{0}\right)
$$


Et par le Lemme 4, on a

$$
\begin{aligned}
& F_{Q}\left(T / R_{0}\right)=J_{Q}\left(T / R_{0}\right) \oplus \\
& \left\langle\left(T / R_{0}\right) /\left(R_{T} / R_{0}\right)-\sum_{\substack{\left|Z / R_{T}\right|=p \\
Z / R_{T} \prec \mathcal{Z}\left(T / R_{T}\right)}}\left(T / R_{0}\right) /\left(Z / R_{0}\right) \mid\left(T, R_{T}\right) \in \Sigma_{Q}(T)\right\rangle
\end{aligned}
$$

donc

$$
\begin{aligned}
& \operatorname{Ind}_{T}^{P} \operatorname{Inf}_{T / R_{0}}^{T} y=\left(P / R_{0}-\sum_{\substack{Z / Z / R_{0} \mid=p \\
Z / R_{0} \prec \mathcal{Z}\left(S_{0} / R_{0}\right)}} P / Z\right) \in J_{Q}(P)+ \\
& \left\langle P / R_{T}-\sum_{\substack{\left|Z / R_{T}\right|=p \\
Z / R_{T} \prec \mathcal{Z}\left(T / R_{T}\right)}} P / Z, \operatorname{avec}\left|R_{T} / R_{0}\right|=p \text { et }\left(T, R_{T}\right) \in \Sigma_{Q}(T)\right\rangle .
\end{aligned}
$$

On remarque qu'on est passé d'une section $\left(S_{0}, R_{0}\right)$ de $P$ telle que $\left(S_{0} / R_{0}\right) \simeq$ $Q$ à une autre section $\left(T, R_{T}\right)$ de $P$ telle que $\left(T / R_{T}\right) \simeq Q$, et $\left|R_{T} / R_{0}\right|=p$. Comme $|P / T|=p^{n}$, alors par hypothèse de récurrence on a

$$
\begin{aligned}
& \left(P / R_{T}-\sum_{\substack{Z / R_{T} \mid=p \\
Z / R_{T} \prec \mathcal{Z}\left(T / R_{T}\right)}} P / Z\right) \in \\
& J_{Q}(P)+\left\langle P / R-\sum_{\substack{|Z / R|=p \\
Z / R \prec \mathcal{Z}(Q)}} P / Z \mid\left(N_{P}(R) / R\right) \cong Q\right\rangle .
\end{aligned}
$$

Ainsi la section $\left(S_{0}, R_{0}\right)$ vérifie la propriété $\mathcal{P}$, et on a

$$
F_{Q}(P)=J_{Q}(P)+\left\langle P / R-\sum_{\substack{|Z / R|=p \\ Z / R \prec \mathcal{Z}\left(N_{P}(R) / R\right)}} P / Z \mid\left(N_{P}(R) / R\right) \cong Q\right\rangle .
$$

On en déduit alors que

$\operatorname{dim}_{k} S_{Q, k}(P)$

$\leq \mid\left\{\right.$ classes de conjugaison de sous-groupes $R$ de $\left.P \mid\left(N_{P}(R) / R\right) \cong Q\right\} \mid$.

Finalement montrons que dans $F_{Q}(P)$ :

$$
J_{Q}(P) \cap\left\langle P / R-\sum_{\substack{|Z / R|=p \\ Z / R \prec \mathcal{Z}(P / R)}} P / Z \mid(P, R) \in \Sigma_{Q}(P)\right\rangle=\{0\} .
$$


En effet: soit

$$
x=\sum_{(P, R) \in \Sigma_{Q}(P)} \lambda_{R}\left(P / R-\sum_{\substack{|Z / R|=p \\ Z / R \prec \mathcal{Z}(P / R)}} P / Z\right) \in J_{Q}(P)
$$

vérifions que

$$
\lambda_{R}=0, \forall(P, R) \in \Sigma_{Q}(P) .
$$

Fixons une section $(P, R) \in \Sigma_{Q}(P)$, et appliquons le foncteur $\operatorname{Def}_{P / R}^{P}$ à $x$. On a donc

$$
\operatorname{Def}_{P / R}^{P}(x)=0, \text { dans } k \otimes_{\mathbb{Z}} B(P / R)
$$

i.e.,

$$
\begin{aligned}
0 & =\lambda_{R}\left[(P / R) /(R / R)-\sum_{\substack{|Z / R|=p \\
Z / R \prec \mathcal{Z}(P / R)}}(P / R) /(Z \cdot R / R)\right] \\
& +\sum_{\substack{\left(P, R^{\prime}\right) \in \Sigma_{Q}(P) \\
R^{\prime} \neq R}} \lambda_{R^{\prime}}\left[(P / R) /\left(R^{\prime} \cdot R / R\right)-\sum_{\substack{\left|Z^{\prime} / R^{\prime}\right|=p \\
Z^{\prime} / R^{\prime} \prec \mathcal{Z}(P / R)}}(P / R) /\left(Z^{\prime} \cdot R / R\right)\right] .
\end{aligned}
$$

Dans cette égalité le terme $(P / R) /(R / R)$ est unique, donc $\lambda_{R}=0$. Comme la section $(P, R)$ est arbitraire dans $\Sigma_{Q}(P)$, on obtient $x=0$. Par suite

$$
J_{Q}(P) \cap\left\langle P / R-\sum_{\substack{|Z / R|=p \\ Z / R \prec \mathcal{Z}(P / R)}} P / Z \mid(P, R) \in \Sigma_{Q}(P)\right\rangle=\{0\} .
$$

Ainsi

$$
\operatorname{dim}_{k} S_{Q, k}(P) \geq \mid\{\text { sous-groupes } R \text { de } P \mid(P / R) \cong Q\} \mid \text {. }
$$

Corollaire 1. Soient $Q$ et $P$ deux p-groupes finis, tel que $Q$ est d'ordre au moins $p^{2}$. Si tous les sous-groupes de $P$ sont normaux (par exemple si $P$ est abélien), alors $\operatorname{dim}_{k} S_{Q, k}(P)$ est égale au nombre de sous-groupes $R$ de $P$ tels que $P / R \simeq Q$.

Preuve. Si tous les sous-groupes de $P$ sont normaux, alors par la Proposition 3 on a

$$
F_{Q}(P)=J_{Q}(P) \oplus\left\langle P / R-\sum_{\substack{|Z / R|=p \\ Z / R \prec \mathcal{Z}(P / R)}} P / Z \mid(P, R) \in \Sigma_{Q}(P)\right\rangle
$$


et on conclut

$$
\operatorname{dim}_{k} S_{Q, k}(P)=\mid\{\text { sous-groupes } R \text { de } P \mid(P / R) \cong Q\} \mid .
$$

2.2. Le cas des foncteurs simples $S_{1, k}$. D'après ([1], 7.2.1), $\operatorname{dim}_{k} S_{1, k}(P)$ est égale au rang de la forme bilinéaire sur $k \otimes_{\mathbb{Z}} B(P)$ à valeurs dans $k$ qui au couple $(P / U, P / V)$ associe

$$
\langle P / U, P / V\rangle_{P}=|U \backslash P / V| .
$$

Lemme 5. Soit $P$ un p-groupe fini d'ordre $p^{2}$, alors

$$
k \otimes_{\mathbb{Z}} B(P)=J_{1, k}(P)+\langle P / P, P / R \text { avec }|P / R|=p\rangle .
$$

Preuve. On a

$$
k \otimes_{\mathbb{Z}} B(P)=\langle P / P, P / R \text { avec }|P / R|=p, P / 1\rangle .
$$

Soit

$$
x=P / 1-\sum_{\substack{R \prec P \\|P / R|=p}} P / R .
$$

Vérifions que pour tout sous-groupe $K$ de $P$ on a $\langle P / K, x\rangle=0$, i.e.,

$$
|K \backslash P / 1|-\sum_{\substack{R \prec P \\|P / R|=p}}|K \backslash P / R|=0 .
$$

Il y a trois cas à envisager:

Cas 1 . Si $K=1$, alors

$$
\begin{aligned}
|1 \backslash P / 1|-\sum_{\substack{R \prec P \\
|P / R|=p}}|1 \backslash P / R| & =|P|-\sum_{\substack{R \prec P \\
|P / R|=p}}|P / R| \\
& =0 \text { dans } k .
\end{aligned}
$$

Cas 2. Si $K=P$, alors

$$
\begin{aligned}
|P \backslash P / 1|-\sum_{\substack{R \prec P \\
|P / R|=p}}|P \backslash P / R| & =1-\mid\{R \prec P \text { avec }|P / R|=p\} \mid \\
& =0 \text { dans } k .
\end{aligned}
$$


Cas 3. Si $K=R_{0}$ avec $\left|P / R_{0}\right|=p$, alors on a

$$
\begin{aligned}
\left|R_{0} \backslash P / 1\right|-\sum_{\substack{R \prec P \\
|P / R|=p}}\left|R_{0} \backslash P / R\right| & =\left|P / R_{0}\right|-\sum_{\substack{R \prec P \\
|P / R|=p}}\left|P /\left(R_{0} \cdot R\right)\right| \\
& =-\sum_{\substack{R \prec P \\
|P / R|=p \\
R \neq R_{0}}}|P / P| \\
& =0 \text { dans } k .
\end{aligned}
$$

Donc $x \in J_{1, k}(P)$, par suite

$$
P / 1 \in\left(J_{1, k}(P)+\langle P / P, P / R \text { avec }|P / R|=p\rangle\right)
$$

d'où le lemme.

Proposition 4. Soit $P$ un p-groupe fini, alors

$$
\begin{aligned}
k \otimes_{\mathbb{Z}} B(P) & =J_{1, k}(P)+\left\langle P / P, P / R \text { avec }\left|N_{P}(R) / R\right|=p\right\rangle \\
& \supseteq J_{1, k}(P) \oplus\langle P / P, P / M \text { avec }|P / M|=p\rangle
\end{aligned}
$$

et on a

$$
\begin{aligned}
& \mid\{\text { sous-groupes } R \text { de } P \text { tels que }|P / R| \leq p\} \mid \\
& \leq \operatorname{dim}_{k} S_{1, k}(P) \\
& \leq \mid\{\text { classes de conjugaison de sous-groupes } R \text { de } P \\
& \text { tels que } \left.\left|N_{P}(R) / R\right|=p\right\} \mid .
\end{aligned}
$$

Preuve.

- On sait que

$$
k \otimes_{\mathbb{Z}} B(P)=J_{1, k}(P)+\left\langle P / P, P / R \text { avec }\left|N_{P}(R) / R\right|=p\right\rangle .
$$

En effet, soit $P / R_{0}$ un élément de $k \otimes_{\mathbb{Z}} B(P)$, montrons par récurrence sur l'indice $\left[P: R_{0}\right]$, que la section $\left(P, R_{0}\right)$ vérifie la propriété $\mathcal{P}$ suivante:

$$
P / R_{0} \in\left(J_{1, k}(P)+\left\langle P / P, P / R \text { avec }\left|N_{P}(R) / R\right|=p\right\rangle\right) .
$$

Si $\left|P / R_{0}\right|=1$, i.e., $R_{0}=P$, alors la propriété $\mathcal{P}$ est vérifiée.

Soit $P / R_{0}$ un élément de $k \otimes_{\mathbb{Z}} B(P)$ tel que $\left|P / R_{0}\right|=p^{n+1}$. Supposons que la propriété $\mathcal{P}$ est vérifiée pour les sections $(P, R)$ telles que l'indice $|P / R|$ est inférieur à $p^{n}$, et montrons que la section $\left(P, R_{0}\right)$ vérifie aussi la propriété $\mathcal{P}$ :

$\mathrm{Si}\left|N_{P}\left(R_{0}\right) / R_{0}\right| \leq p$, alors

$$
P / R_{0} \in\left(J_{1, k}(P)+\left\langle P / P, P / R \text { avec }\left|N_{P}(R) / R\right|=p\right\rangle\right) .
$$


Et si $\left|N_{P}\left(R_{0}\right) / R\right| \geq p^{2}$, alors il existe un sous-groupe $T$ de $P$ tel que $R_{0}$ est normal dans $T$ et $\left|T / R_{0}\right|=p^{2}$. D'après le Lemme 5 on a

$$
\begin{aligned}
x= & \left(T / R_{0}\right) /\left(R_{0} / R_{0}\right) \in\left(J_{1, k}\left(T / R_{0}\right)\right. \\
& \left.+\left\langle\left(T / R_{0}\right) /\left(T / R_{0}\right),\left(T / R_{0}\right) /\left(M / R_{0}\right) \text { avec }|T / M|=p\right\rangle\right)
\end{aligned}
$$

donc

$\operatorname{Ind}_{T}^{P} \operatorname{Inf}_{T / R_{0}}^{T} x=P / R_{0} \in\left(J_{1, k}(P)+\langle P / T, P / M\right.$ avec $\left.|T / M|=p\rangle\right)$.

On remarque qu'on est passé d'une section $\left(T, R_{0}\right)$ de $P$ telle que $\left|T / R_{0}\right|=p^{2}$ à deux autres sections de $P:(P, M)$ et $(P, T)$ telles que $\left|M / R_{0}\right|=p$ et $|T / M|=p$. Comme $|P / M|=p^{n}$ et $|P / T|=p^{n-1}$, alors par hypothèse de récurrence on a

$$
P / M \in\left(J_{1, k}(P)+\left\langle P / P, P / R \operatorname{avec}\left|N_{P}(R) / R\right|=p\right\rangle\right)
$$

et

$$
P / T \in\left(J_{1, k}(P)+\left\langle P / P, P / R \text { avec }\left|N_{P}(R) / R\right|=p\right\rangle\right) .
$$

Ainsi la section $\left(P, R_{0}\right)$ vérifie la propriété $\mathcal{P}$, et on a

$$
k \otimes_{\mathbb{Z}} B(P)=J_{1, k}(P)+\left\langle P / P, P / R \text { avec }\left|N_{P}(R) / R\right|=p\right\rangle .
$$

Comme $S_{1, k}(P)=k \otimes_{\mathbb{Z}} B(P) / J_{1, k}(P)$, il en résulte que

$$
\operatorname{dim}_{k} S_{1, k}(P)
$$

$\leq \mid\{$ classes de conjugaison de sous-groupes $R$ de $P$

$$
\text { tels que } \left.\left|N_{P}(R) / R\right|=p\right\} \mid \text {. }
$$

- Dans $k \otimes_{\mathbb{Z}} B(P)$, on a

$$
J_{1, k}(P) \cap\langle P / P, P / M \text { avec }|P / M|=p\rangle=\{0\} .
$$

Soit

$$
\left(x=\lambda_{P} P / P+\sum_{\substack{M \prec P \\|P / M|=p}} \lambda_{M} P / M\right) \in J_{1, k}(P) .
$$

Alors, pour tout sous-groupe $K$ de $P$ on a $\langle P / K, x\rangle=0$, i.e.,

$$
\lambda_{P}|K \backslash P / P|+\sum_{\substack{M \prec P \\|P / M|=p}} \lambda_{M}|K \backslash P / M|=0 .
$$

En particulier pour $K=1$, l'égalité (\$) devient

$$
\lambda_{P}|1 \backslash P / P|=0 \text {. }
$$


Donc $\lambda_{P}=0$, et par suite

$$
x=\sum_{\substack{M \prec P \\|P / M|=p}} \lambda_{M} \cdot P / M \in J_{1, k}(P) .
$$

Appliquons l'égalité (\$) pour le sous-groupe $K=P$, d'où

$\left(\boldsymbol{A}_{1}\right)$

$$
\sum_{\substack{M \prec P \\|P / M|=p}} \lambda_{M}=0 .
$$

Fixons un sous-groupe $M_{0}$ de $P$ tel que $\left|P / M_{0}\right|=p$, alors en appliquant l'égalité (\$) pour le sous-groupe $K=M_{0}$, on obtient

$\left(\boldsymbol{\top}_{2}\right)$

$$
\sum_{\substack{M \prec P \\ M \neq M_{0} \\|P / M|=p}} \lambda_{M}=0 .
$$

En faisant la différence $\left(\boldsymbol{\$}_{1}-\mathbf{Q}_{2}\right)$, on tire: $\lambda_{M_{0}}=0$. Puisque $M_{0}$ est arbitraire, alors $x=0$, et par suite dans $k \otimes_{\mathbb{Z}} B(P)$ on a

$$
J_{1, k}(P) \cap\langle P / P, P / M \text { avec }|P / M|=p\rangle=\{0\} .
$$

Il vient donc

$$
\operatorname{dim}_{k} S_{1, k}(P) \geq \mid\{\text { sous-groupes } R \text { de } P \text { tels que }|P / R| \leq p\} \mid .
$$

Ce qui achève la démonstration de la proposition.

Corollaire 2. Soit $P$ un p-groupe fini. Si tous les sous-groupes de $P$ sont normaux (par exemple si $P$ est abélien), alors $\operatorname{dim}_{k} S_{1, k}(P)$ est égale au nombre de sous-groupes $R$ de $P$ tels que $|P / R| \leq p$.

Preuve. Dans le cas où tous les sous-groupes de $P$ sont normaux, alors par la Proposition 4 on a

$$
k \otimes_{\mathbb{Z}} B(P)=J_{1, k}(P) \oplus\langle P / P, P / M \text { avec }|P / M|=p\rangle
$$

ce qui donne

$$
\operatorname{dim}_{k} S_{1, k}(P)=\mid\{\text { sous-groupes } R \text { de } P \text { tels que }|P / R| \leq p\} \mid .
$$

Acknowledgements. I would like to thank Serge Bouc for perseverance in answering my queries as well as for useful comments. 


\section{References}

[1] S. Bouc, Foncteurs d'ensembles munis d'une double action, J. Algebra, 183(3) (1996), 664-736, MR 1401673 (97j:20017), Zbl 0858.19001.

[2] S. Bouc and J. Thévenaz, The group of endo-permutation modules, Invent. Math., 139 (2000)(2), 275-349, MR 1738450 (2001i:20023), Zbl 0954.20002.

UFR de Mathématiques

Université Paris 7, Case 7012

2, Place Jussieu, 75251 Paris Cedex 05

FRANCE

E-mail address: bourizk@ccr.jussieu.fr 